\title{
Long period variables in the globular cluster 47 Tuc: Radial velocity variations
}

\author{
T. Lebzelter ${ }^{1}$, P. R. Wood ${ }^{2}$, K. H. Hinkle ${ }^{3}$, R. R. Joyce ${ }^{3}$, and F. C. Fekel ${ }^{4}$ \\ ${ }^{1}$ Institute for Astronomy (IfA), University of Vienna, Türkenschanzstrasse 17, 1180 Vienna, Austria \\ e-mail: lebzelter@astro.univie.ac.at \\ 2 Research School for Astronomy \& Astrophysics, Mount Stromlo Observatory, Weston, ACT 2611, Australia \\ 3 National Optical Astronomy Observatory ${ }^{\star}$, PO Box 26732, Tucson, AZ 85726, USA \\ ${ }^{4}$ Center of Excellence in Information Systems, Tennessee State University, Nashville, TN 37203-3401, USA
}

Received 29 January 2004 / Accepted 31 October 2004

\begin{abstract}
We present near infrared velocity curves for 12 long period variables (LPVs) in the globular cluster 47 Tuc (NGC 104). New light curves are also presented for these variables. Results are compared with the period-luminosity sequences occupied by the LPVs in the LMC. Sequence C variables (fundamental mode pulsators) have larger velocity amplitudes than sequence B variables (first overtone pulsators). We show that, at similar luminosities, higher mass loss rates are associated with larger pulsation amplitudes. One variable (V18) does not fit the normal period luminosity sequences and it has an unusually large amount of circumstellar dust, suggesting that it has recently undergone a thermal pulse on the AGB. Finally, we report the discovery of three new long period variable stars in the cluster core, all previously found to have a large infrared excess.
\end{abstract}

Key words. stars: late-type - stars: AGB and post-AGB - stars: evolution

\section{Introduction}

In recent years, there have been significant advances in our understanding of pulsation in long period variable stars (LPVs). Particularly important is the discovery of multiple periodluminosity relations for AGB stars in the LMC and the interpretation of all but one of these relations in terms of radial pulsation in low order modes (Wood et al. 1999). Another advance is the realization of the importance of pulsation in the production of mass loss from red giants (e.g. Wood 1979; Bowen \& Willson 1991; Höfner et al. 1996).

One problem with the observational study of pulsation and mass loss in red giants is that nearly all the stars studied so far are field stars. This means that we have no way of directly determining the initial or current stellar mass. Any relations that are found for the field stars will be broadened by the range in mass (age) and metallicity existing among the stars. For Galactic stars, but not LMC stars, we have the additional problem that the luminosity (distance) is generally poorly known. As a result of these uncertainties, precise comparison of observed periods, luminosities, amplitudes and mass loss rates with theoretical calculations is complicated. A way around these problems is to observe pulsating red giant stars in star clusters where the giants have a common (initial) mass,

* Operated by the Association of Universities for Research in Astronomy, Inc. under cooperative agreement with the National Science Foundation. composition and distance. We can then readily compare theoretical and observed period-luminosity relations, and we can see how the mass loss rate depends on pulsation properties and quantities such as mass, luminosity and metallicity.

The driving of mass loss by pulsation is a complicated process. Levitation of the stellar atmosphere caused by the pulsation leads to the formation above the photosphere of a cool and dense environment where dust grains form and grow efficiently. Radiation pressure on dust grains then combines with momentum in shock waves to drive the mass loss (e.g. Wood 1979; Bowen \& Willson 1991; Höfner et al. 1996). It is found that larger pulsation velocity amplitudes and higher luminosities drive a higher mass loss rate. One of the main purposes of this study is to observe the pulsation velocity amplitudes in a good sample of red variables in a star cluster so that a comparison of mass loss rate with pulsation velocity amplitude (and luminosity) can be made.

The pulsation velocities of LPVs are best measured in the near infrared since optical absorption lines measure velocities in the outer layers only. These optical velocities are nearly always directed inward relative to the stellar center-of-mass (e.g. Wood 1979). The near-infrared lines reveal the deeper, larger amplitude pulsation. Monitoring of the velocity variations in field variables using infrared $(1.6 \mu \mathrm{m}) \mathrm{CO}$ lines has been carried out by Hinkle (1978), Hinkle et al. $(1982,1997)$ and Lebzelter et al. (2000). These observations revealed velocity curves with amplitudes between 3 and $30 \mathrm{~km} \mathrm{~s}^{-1}$. 
The velocity curves of Miras are sawtooth-shaped and of large amplitude, with phases of line doubling around maximum light. The typical velocity curves of semiregular variables (SRVs) are of smaller amplitude than in Miras and they are rather sinusoidal (i.e. continuous with no line doubling), although they can reflect the partly irregular behaviour seen in the light curves. A summary is given in Lebzelter \& Hinkle (2002).

The globular cluster 47 Tuc (NGC 104) was chosen for this study since it contains the richest known collection of long period variables (LPVs). Properties of this cluster from the literature are summarized in Sect. 2. Along its giant branch, four Miras and more than 10 semiregular and irregular variables have been detected (Sawyer-Hogg 1973; Lloyd-Evans 1974). Several infrared searches for indications of mass loss from giant branch stars have also been reported (see Sect. 3).

\section{47 Tuc and its variables}

47 Tuc is a prototype "metal-rich" globular cluster as well as being one of the closest. Recently, Gratton et al. (2003) presented a very detailed study on the fundamental parameters of 47 Tuc. They derive a metallicity of $[\mathrm{Fe} / \mathrm{H}]=-0.66 \pm 0.04$ on the Carretta \& Gratton (1997) scale, which is in agreement with the findings of several other groups (see references in Gratton et al.). A metallicity based on the Zinn \& West (1984) scale is given by e.g. Briley et al. (1995) with $[\mathrm{Fe} / \mathrm{H}]=-0.76$.

An accurate distance to the cluster is still a matter of some debate. Values in the literature for the distance modulus of 47 Tuc scatter by about \pm 0.2 mag (for an overview see Gratton et al. 2003). Gratton et al. (2003) find a distance modulus of $(m-M)_{V}=13.50 \pm 0.08$ from main sequence fitting. All studies agree that there is very little reddening towards 47 Tuc $(E(B-V)=0.024 \pm 0.05)$. Based on their estimates for the distance and metallicity Gratton et al. (2003) derived an age of 47 Tuc of $11.2 \pm 1.1$ Gyr. Hesser et al. (1987) derived a turnoff mass of $0.9 M_{\odot}$, but used an age estimate of 13.5 Gyr. However, with the age from Gratton et al. and the more recent theoretical isochrones from Bertelli et al. (1994) we again come to a turnoff mass between 0.86 and $0.9 M_{\odot}$.

A large number of photometric measurements in the blue, visual and near- and mid-infrared (Lee 1977; Frogel 1983; Montegriffo et al. 1995; Origlia et al. 1997; Ramdani \& Jorissen 2001) establish a very accurate color-magnitude diagram of this cluster showing a well defined AGB. Luminosities and surface temperatures have been derived for the AGB stars (e.g. Whitelock 1986).

Light curves of the LPVs V1 to $\mathrm{V}^{1}$ were measured by Arp et al. (1963). Several additional variables were detected by Lloyd-Evans \& Menzies (1973). A further study, including the variables V3 to V7, V11(=W12), V13 and V18, was presented by Fox (1982). The light variability of these stars will be further discussed in Sect. 4.1.

\footnotetext{
1 Throughout this paper we use the nomenclature from the catalogue of globular cluster variables by Clement et al. (2001).
}

\section{Measurements of mass loss in 47 Tuc}

There have been many attempts to measure the mass loss from AGB stars in globular clusters. Mass loss at the end of the red giant branch (RGB, also called the first giant branch or FGB) phase has already been proposed to explain the observed gaps in the horizontal branch of globular clusters (Soker et al. 2001; Schröder \& Sedlmayr 2001). Cohen (1976) proposed the existence of circumstellar shells (and hence mass loss) around first giant branch stars in globular clusters to explain emission components in the $\mathrm{H} \alpha$ line. Bates et al. (1990) summarized different studies on $\mathrm{H} \alpha$ lines of globular cluster stars and found a further indication for circumstellar shells from the $\mathrm{NaD}$ line profiles. Lyons et al. (1996) discussed mass motions in the atmospheres of 63 red giants from five different globular clusters with the help of $\mathrm{H} \alpha$ and $\mathrm{NaD}$ lines. Core shifts of these lines indicate mass flow. The lower luminosity limit for outflow from $\mathrm{H} \alpha$ and $\mathrm{NaD}$ lines is $\log \left(L / L_{\odot}\right)=2.5$ and 2.9 , respectively. Only part of the red giants showed an outflow. Standard deviations in the final masses of white dwarfs in globular clusters of the order of 0.1 mag may indicate a stochastic nature of the mass loss (e.g. see the discussion by Alves et al. 2000).

Not only is mass loss known to be essential in stellar evolution but the mass loss from red giants in globular clusters should result in intracluster gas and dust (Evans et al. 2003). Searches for the intracluster gas have been limited in their results. The low metallicity no doubt plays a role in reducing silicate emission, making dust detection difficult (Frogel \& Elias 1988; Helling et al. 2002). However, findings from AGB stars in the Large Magellanic Cloud (LMC) confirm that low metallicity stars reach high mass loss rates (e.g. Wood et al. 1992; van Loon et al. 1999).

A few recent papers provide more detailed information on circumstellar shells around AGB stars in 47 Tuc. One of the most definitive results is by Ramdani \& Jorissen (2001) who used the ISO satellite to measure the mid-infrared emission around 6 AGB variables in 47 Tuc. Their findings, however, highlight the difficulty of correlating pulsational properties with mass loss. Half of the variables, namely V5, V7 and V15, show no or only marginal $12 \mu$ m-excess while the other three stars, V3, V11 and V18, do have a detectable excess flux at $12 \mu \mathrm{m}$. The excess of V11 is rather small. V3 is a Mira and would be expected to show an excess. But the largest infrared excess was measured for V18, an irregular AGB variable of only modest luminosity. Origlia et al. (2002) suggested that the mass loss from red giant stars is episodic. They found an IR excess for V8 and no excess for V21 (see also Sect. 5.3).

Overall, some indication of the presence or absence of circumstellar material exists for 12 AGB variables in 47 Tuc. A substantial infrared excess in V3, V11 and V18 was detected by Gillett et al. (1988), but these authors did not find indications for an infrared excess in V5 and V13. An excess $V$-band polarization was found in V1 to V4, V6, V8 and V11 by Forte et al. (2002) indicating the existence of circumstellar material around these stars. Glass \& Feast (1973) reported an $L$-band excess in V3. Frogel \& Elias (1988) detected a $10 \mu \mathrm{m}$ excess in the four cluster variables V1, V2, V3 and V4. They calculated total mass loss rates for these objects ranging between 4.6 and 
Table 1. Properties of LPVs in 47 Tuc.

\begin{tabular}{lccccc}
\hline \hline Name & $\alpha(2000)$ & $\delta(2000)$ & $J$ & $K$ & $P[\mathrm{~d}]$ \\
\hline V1 & 002412.4 & -720639 & 7.45 & 6.21 & 221 \\
V2 & 002418.4 & -720759 & 7.52 & 6.29 & 203 \\
V3 & 002515.9 & -720354 & 7.49 & 6.27 & 192 \\
V4 & 002400.3 & -720726 & 7.87 & 6.69 & 165,82 \\
V5 & 002503.7 & -720931 & 8.65 & 7.47 & 50 \\
V6 & 002425.5 & -720630 & 8.54 & 7.43 & 48 \\
V7 & 002520.6 & -720640 & 8.18 & 6.97 & 52 \\
V8 & 002408.3 & -720354 & 7.94 & 6.70 & 155 \\
V11 & 002509.0 & -720217 & 7.91 & 6.71 & $52,160 ?$ \\
V13 & 002258.3 & -720656 & 8.79 & 7.70 & 40 \\
V18 & 002509.2 & -720239 & 8.59 & 7.47 & $83 ?$ \\
V21 & 002350.1 & -720550 & 8.07 & 6.78 & 76 \\
\hline
\end{tabular}

Notes: For V18 and V21 both period determinations are from this study. For V1 we suggest a period of 221 days from our data instead of 212 days listed in the literature. For V5 we favour a period of 50 days (instead of 60 days listed in the Clement et al. catalogue). For V11 see text. All other periods are from the catalogue of Clement et al. (2001). The $J$ and $K$ magnitudes are averages of the maximum and minimum observed values from the following sources: the 2MASS Point Source Catalog; Fox (1982); Menzies \& Whitelock (1985); and Frogel et al. (1988). All magnitudes have been converted to the AAO system using conversions in Allen \& Cragg (1983) and Carpenter (2004).

$12.3 \times 10^{-6} M_{\odot} \mathrm{yr}^{-1}$. A $10 \mu \mathrm{m}$ excess was also detected in $\mathrm{V} 1$ and V3 by Origlia et al. (1997). However their estimated dust mass loss rates are about ten times less than those of Frogel \& Elias (1988).

\section{Observations and results}

We selected 12 long period variables in the globular cluster 47 Tuc for which a period or at least some information on the variability type was given in the catalogue of variable stars in globular clusters published by Clement et al. (2001). These stars are moderately bright infrared sources, with $K \sim 6-8$. Table 1 summarizes the properties of the stars.

Time series of infrared spectra in the $H$ band were obtained in 2001 and 2002 with the 74 inch telescope at Mount Stromlo Observatory, Australia. The NICMASS detector, already successfully used for a preceding program at Kitt Peak (Joyce et al. 1998), was used at the Coudé focus of the telescope. The standard infrared observation technique was used. Spectra of each star were obtained at two different slit positions to allow sky subtraction. Resolution was set to $R=37000$. We achieved a $\mathrm{S} / \mathrm{N}$ ratio of 30 or better. An example spectrum is shown in the left part of Fig. 1 with an identification of some relevant spectral features. The spectral range covered a number of second overtone $\mathrm{CO}$ lines, some $\mathrm{OH}$ lines, and a few metallic lines. When 47 Tuc was visible at Mount Stromlo spectra were obtained approximately once a month. The observing program had an unexpected end when Mount Stromlo Observatory was destroyed by a bush fire in early 2003 . Nevertheless time coverage and sampling of the 47 Tuc AGB variables, combined with a few additional spectra taken in the same wavelength region

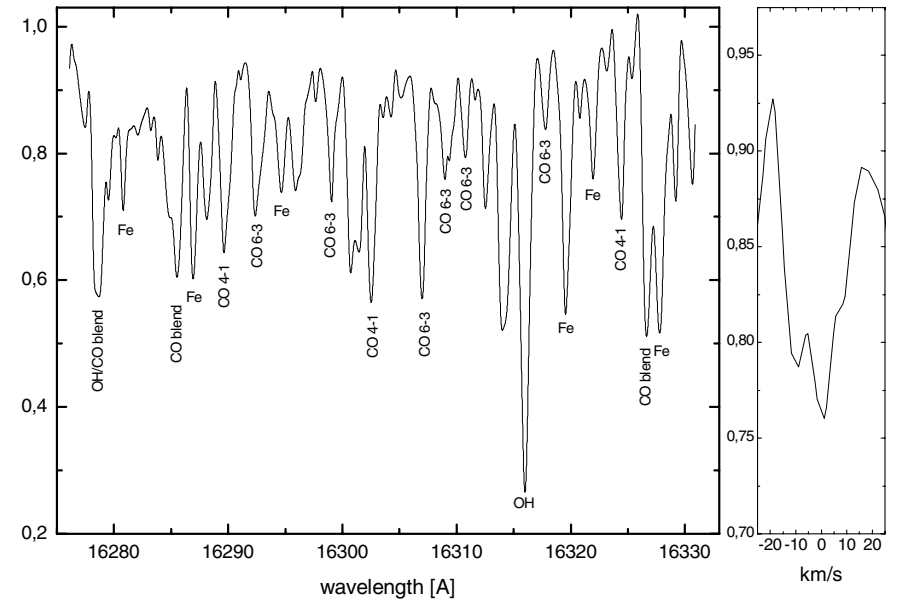

Fig. 1. Left panel: example spectrum of the star V11. Several prominent features are identified. Right panel: example of line doubling (CO 4-1, V3).

with the PHOENIX spectrograph at Gemini South, is sufficient for the investigation presented here.

The bright stars $\alpha$ Cet and $\delta$ Oph have been used as primary velocity standards (Udry et al. 1999). Velocities of the variables were determined by a cross correlation technique, using the IRAF task fxcor. Typical velocity uncertainties, determined from multiple observations of some stars in the same or consecutive nights, were found to be $\sim 0.4 \mathrm{~km} \mathrm{~s}^{-1}$.

To determine proper phases for the velocity curve, photometric measurements of all variables in our sample except V5 (see below) were done as well. Data in the blue and red MACHO filters were obtained with the 50inch telescope at Mount Stromlo (also destroyed in the January 2003 fires). $V$ and $I$ measurements were obtained with ANDICAM at the YALO telescope (before the MACHO dataset), with a few additional measurements from ANDICAM at the CTIO 1.3 m telescope (after the MACHO dataset). The different data sets have been combined using transforms based on about 40 nonvariable cluster stars ranging in $V-I$ between 0.9 and 2.1. The MACHO blue filter has a mean wavelength close to that of the $V$ filter so MACHO blue magnitudes can be reliably transformed to $V$. Long time series are therefore available in the $V$ filter. No obvious phase shift relative to the other filters was noticed, but a conclusive result cannot be drawn from our data. From the $V$ light curves we determined the time of the light maxima, phase zero.

\subsection{Light curves}

Light curves for most of the variables are shown in the upper panels of Figs. 2 to 13. Periods derived from our measurements were in most cases in good agreement with the values from the literature. In our data V4 shows a main period of $\sim 170 \mathrm{~d}$ in agreement with the value of $165 \mathrm{~d}$ from Arp et al. (1963). Fox (1982) found a period of 82 days for V4 and suggested that this star switched between two modes. This type of multimode behaviour has subsequently been found to be common in the light curves of LPVs (e.g. Wood et al. 1999). 


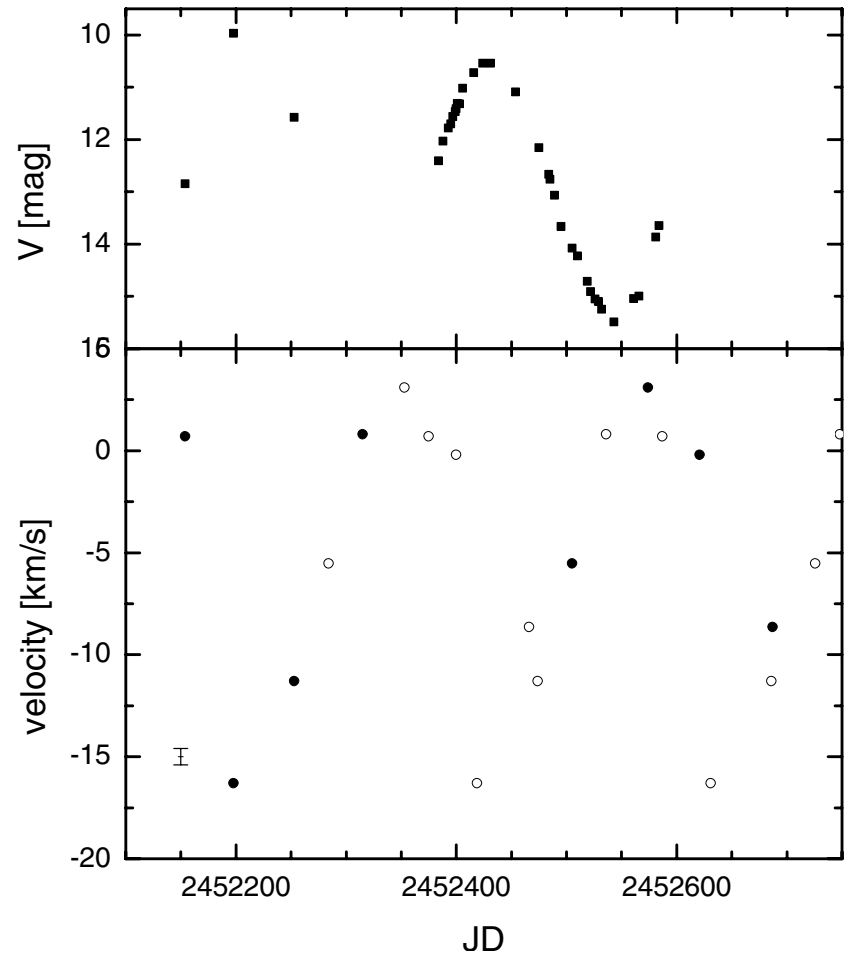

Fig. 2. Light (upper panel) and velocity variations (lower panel) for V1. Filled symbols in the lower panel indicate individual velocity measurements. For a better illustration of the velocity change data are repeated shifted by an integral number of periods forward and backward in time (open symbols). The period listed in Table 1 is used. The typical error bar for the velocity data is indicated.

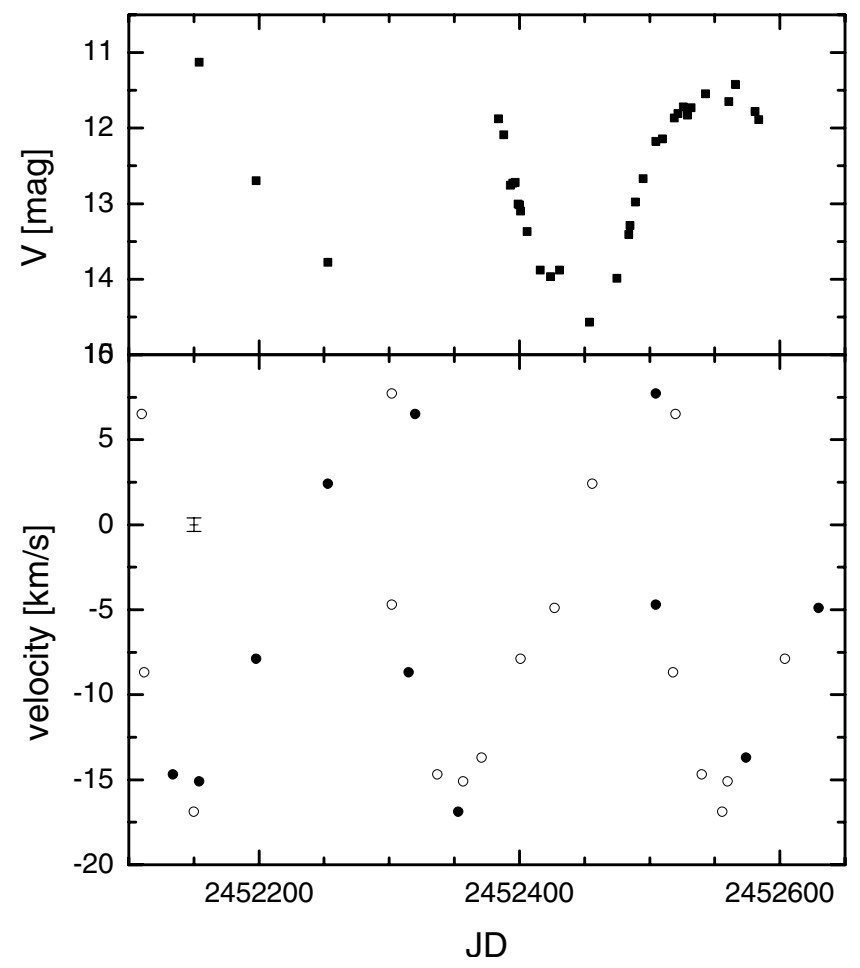

Fig. 3. Same as Fig. 2 for V2.

For V5, we unfortunately do not have sufficient usable data as this star was located in a bad area of the CCD. The

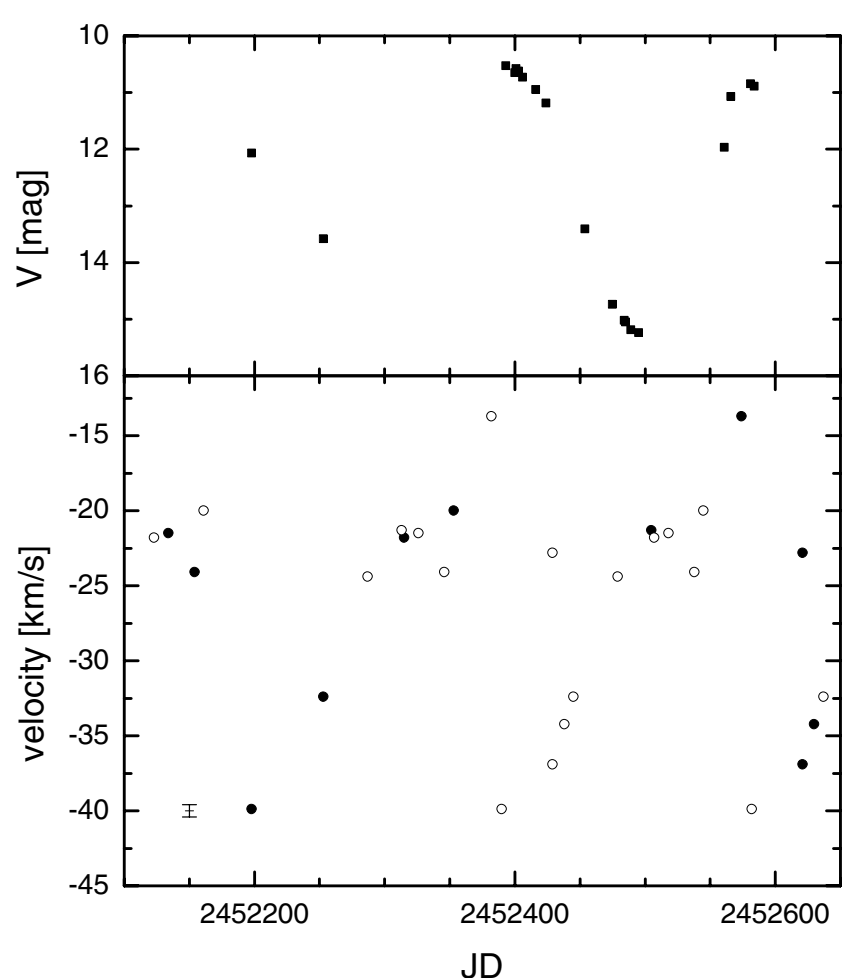

Fig. 4. Same as Fig. 2 for V3.

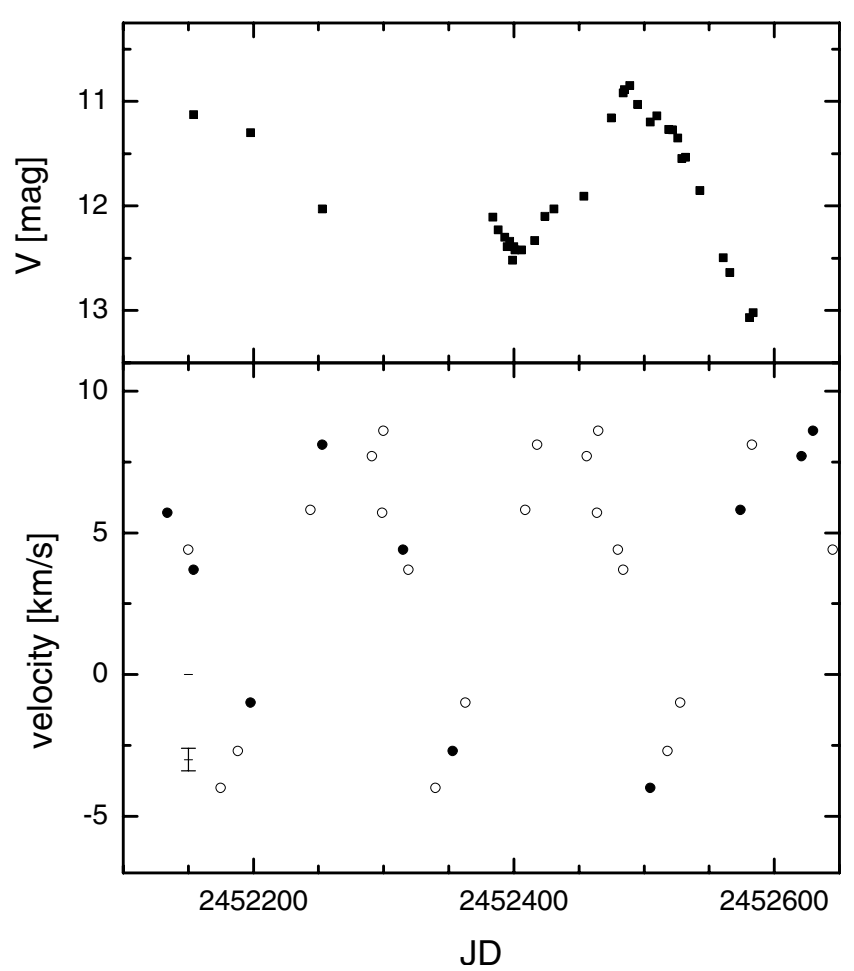

Fig. 5. Same as Fig. 2 for V4.

Sawyer-Hogg value (1973) is 60 days, but different values can be found in the literature, ranging between 29 and 70 days (Fox 1982). From our velocity data we rather favour a period of 50 days. The short periods of 52 and 100 days reported for V11 could not be confirmed in our data. Instead, the star shows some long period variation lasting more than 200 days (Fig. 10). 


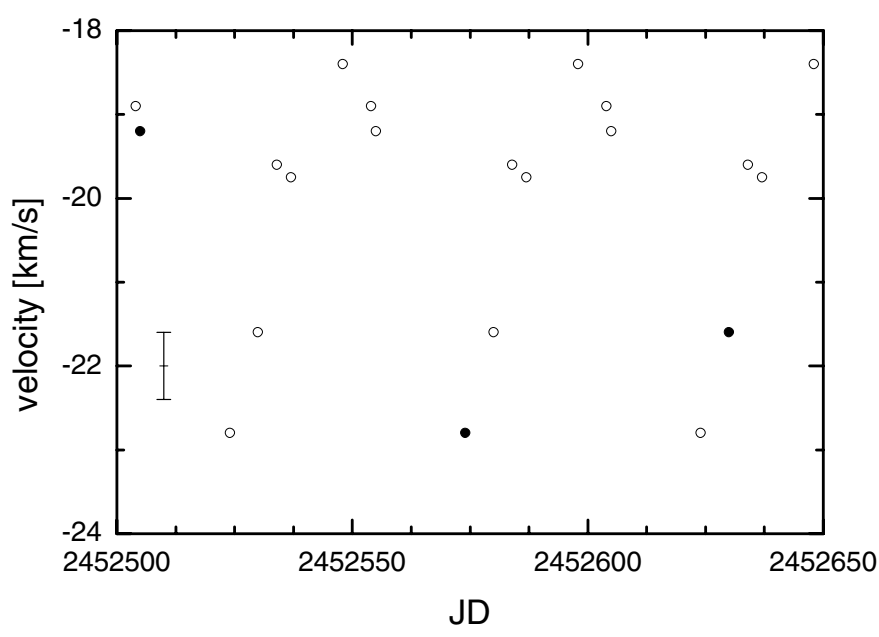

Fig. 6. Velocity change of V5. Only part of the time of monitoring is shown. Symbols as in the lower panel of Fig. 2. No parallel light curve data exist for this star. A typical error bar for the velocity data is indicated.

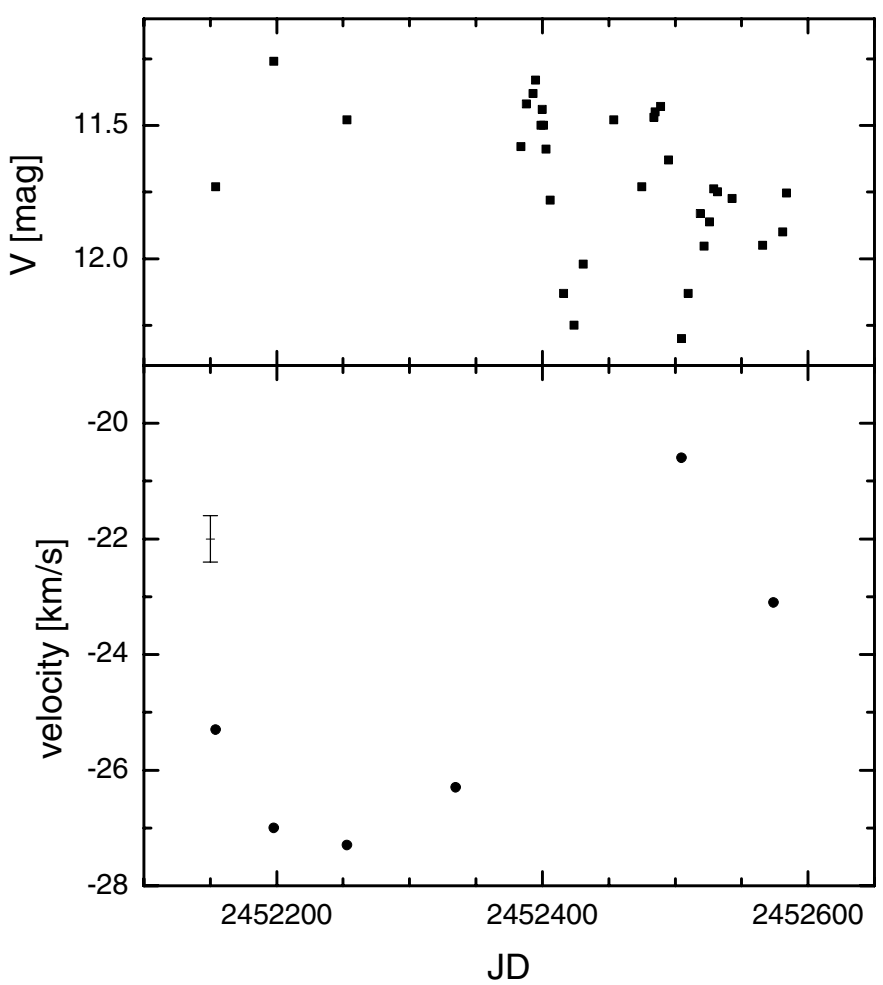

Fig. 7. Light (upper panel) and velocity (lower panel) variations for V6. A typical error bar for the velocity data is indicated.

Compared to the data presented by Fox (1982) the amplitude of the variation is smaller. This star appears to be another multimode pulsator. For V13, there is a hint of the catalogued $\sim 40 \mathrm{~d}$ period in our light curve, but a period of $\sim 90 \mathrm{~d}$ may also be present. Additionally, our light curve suggests a long period (Fig. 11) about 10 times the short period. This star appears to be yet another multimode pulsator.

V18 was reported variable both in the catalogue of SawyerHogg (1973) and by Lloyd Evans (1974), while Fox (1982) found no variability in this star. We found photometric variability with an amplitude of about $0.2 \mathrm{mag}$ in this object (Fig. 12).

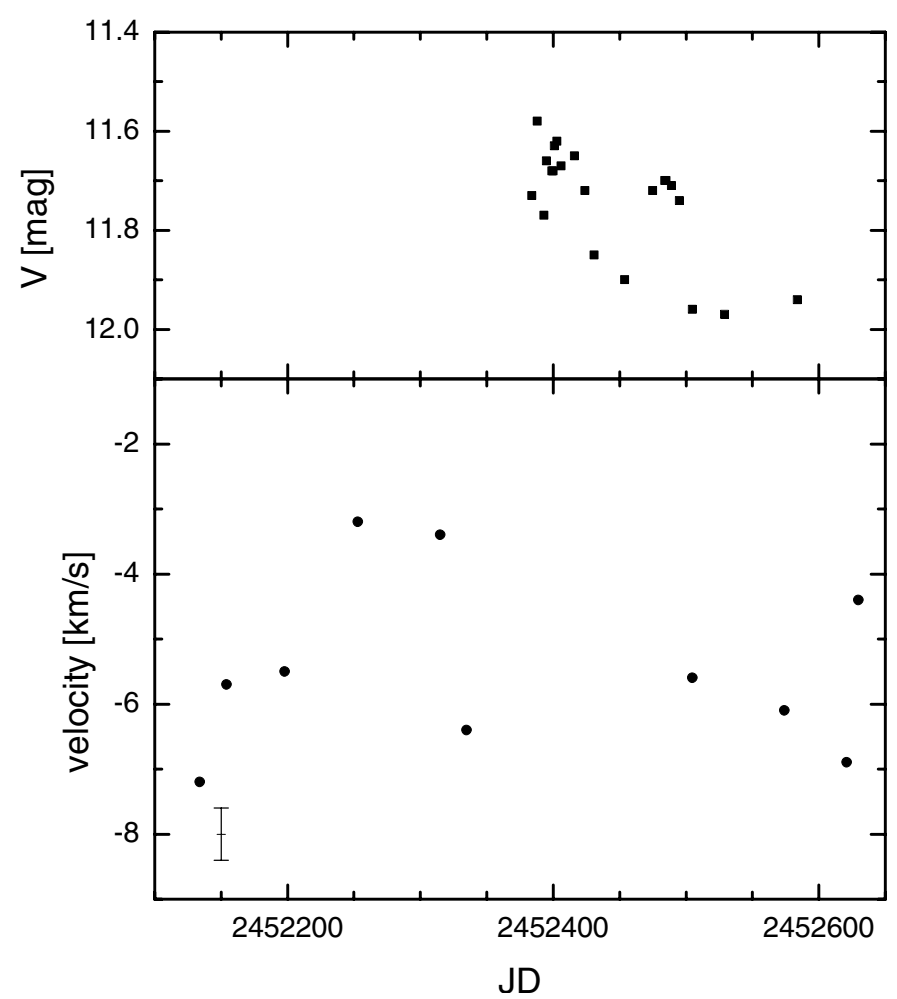

Fig. 8. Same as Fig. 7 for V7.

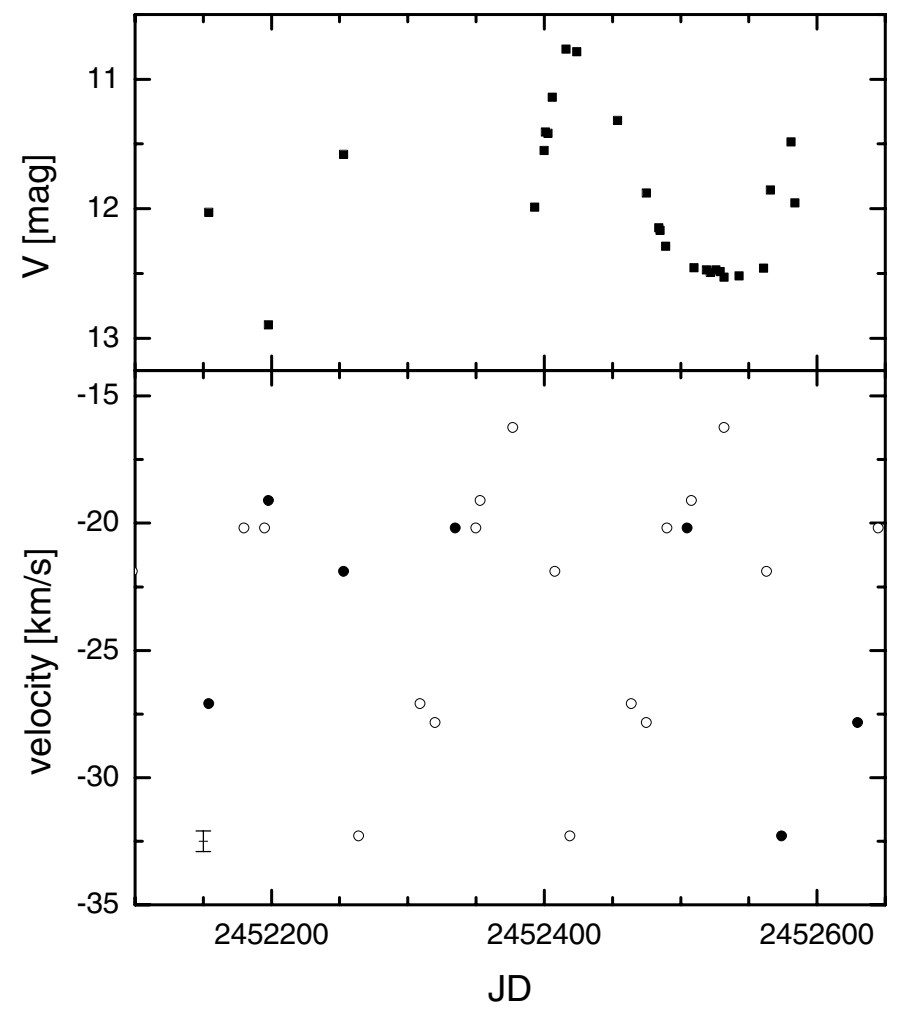

Fig. 9. Same as Fig. 2 for V8.

Our short light curve would suggest a period of about 83 days. For V21, no period determination existed in the literature. Our data set (see Fig. 13) shows a period of 76 days. 


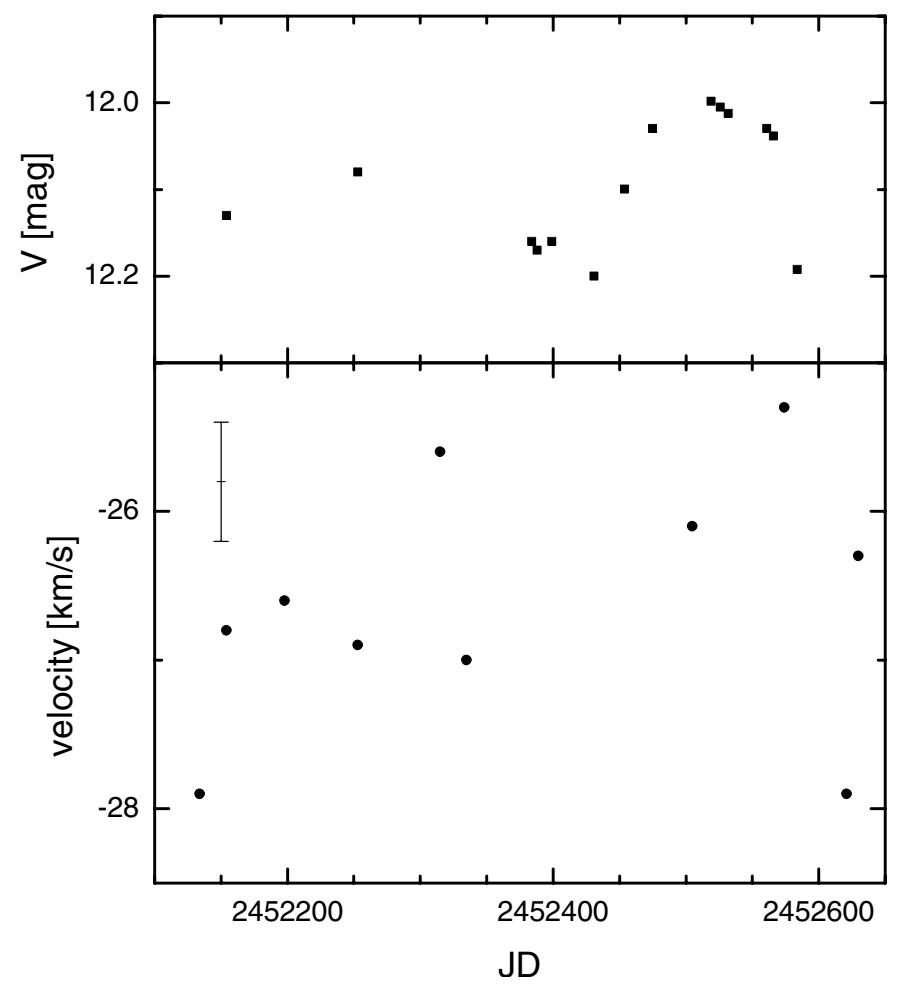

Fig. 10. Same as Fig. 7 for V11.

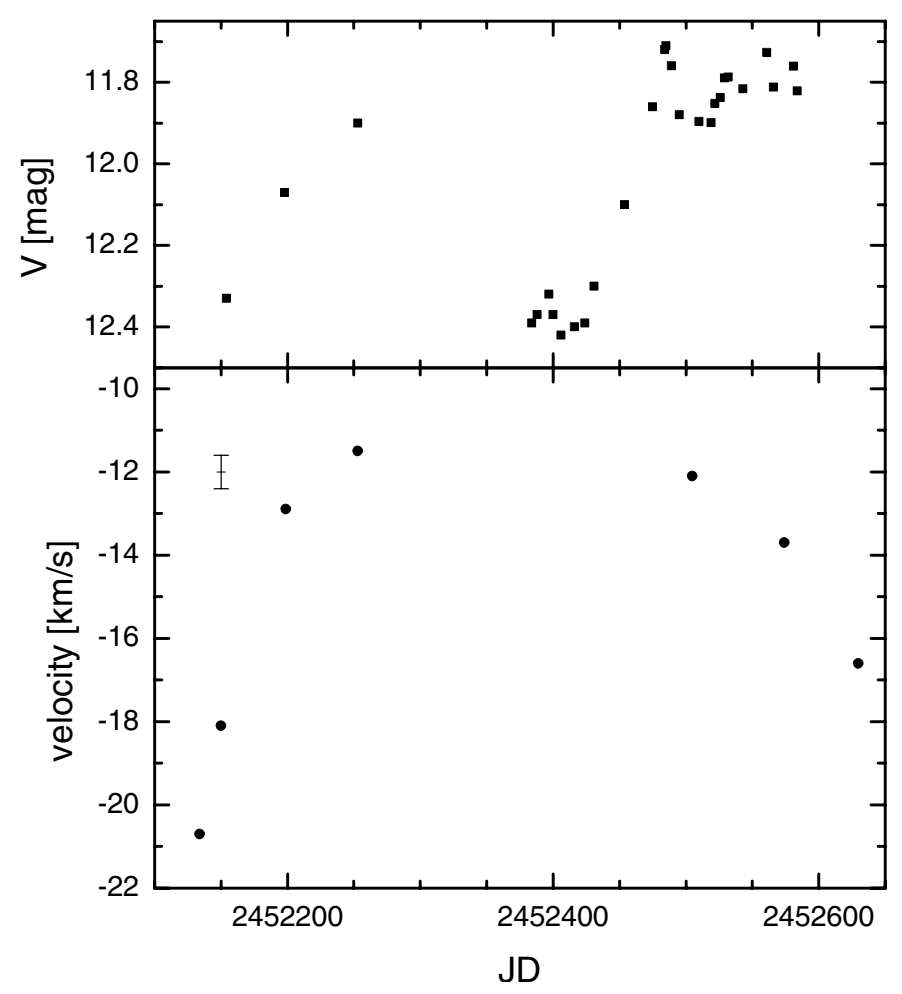

Fig. 11. Same as Fig. 7 for V13.

\subsection{Velocity curves}

Velocity curves were determined for all 12 stars of our sample. All stars show at least some velocity variability above our detection threshold. Line doubling was detected in three stars of



Fig. 12. Same as Fig. 7 for V18.

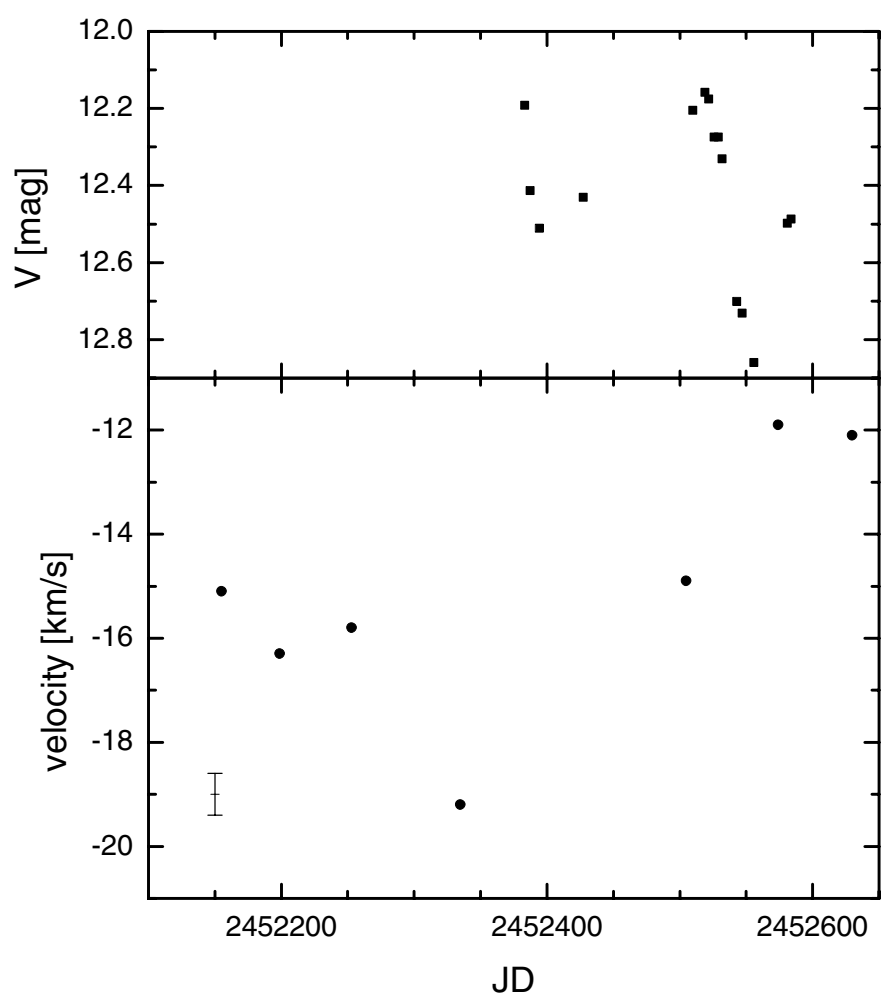

Fig. 13. Same as Fig. 7 for V21.

our sample (Table 2). An example of line doubling is shown in the right part of Fig. 1.

The velocity curves we derived are shown in the lower parts of Figs. 2 to 13 together with the corresponding light change. The velocity curves can be roughly separated into three 
Table 2. Data on the AGB variables in 47 Tuc.

\begin{tabular}{lccl}
\hline \hline Name & $\begin{array}{c}\text { Velocity amplitude } \\
{\left[\mathrm{km} \mathrm{s}^{-1}\right]}\end{array}$ & $\begin{array}{c}\text { Variability } \\
\text { type }\end{array}$ & Comments \\
\hline V1 & 20 & Mira-like & Line doubling \\
V2 & 23 & Mira-like & Line doubling \\
V3 & 22 & Mira-like & Line doubling \\
V4 & 18 & Mira-like & Double-period \\
V5 & 8 & Regular & \\
V6 & 7 & Semiregular & \\
V7 & 4 & Semiregular & \\
V8 & 16 & Mira-like & \\
V11 & 4 & Irregular & \\
V13 & 12 & Long period & Double-period \\
V18 & 5 & Irregular & \\
V21 & 7 & Long period & \\
\hline
\end{tabular}

groups: V1, V2, V3, V4, and V8 show velocity curves very similar in shape to those typical of Miras found in the solar neighborhood (Lebzelter \& Hinkle 2001). The velocity amplitude of V1, V2 and V3 are all similar to nearby Miras, too. The other two stars show a similar shape but a clearly smaller amplitude. While we confirm the Mira-like nature of these five variables (e.g. noted by Whitelock 1986), we note that the smaller velocity amplitude of the latter two stars is also accompanied by a smaller light amplitude. The second group of variables, consisting of V5, V6 and V7 is comparable to local semiregular variables (Lebzelter \& Hinkle 2002). Their amplitudes are much smaller than in the first group. V5 shows a very regular light change, while the other two objects are obviously not strictly periodic, in agreement with the semiregular nature of their light curves. The third group consists of V11, V13, V18, and V21. In all four of these stars, there is no obvious correlation between the light change and the velocity variations. In the case of V13, it cannot be ruled out that variations occur on a time scale similar to the short or even the long period (see above). For V11 our observations do not allow a clear picture of the light change, so a correlation is not possible. On the other hand, V18 and V21 do show a rather well defined light change, but velocity variability occurs on a different time scale. The reason for that is not clear and longer spectroscopic and photometric time series will be needed to understand this phenomenon. Table 2 summarizes the results on the velocity variations in the 47 Tuc AGB variables. The table gives the total velocity amplitude, the characteristics of the velocity curve and comments on the occurrence of line doubling.

\section{Discussion}

\subsection{Pulsation and stellar evolution}

Observations from the Magellanic Clouds indicate that AGB variables are found on four distinct period-luminosity relations (e.g. Wood 2000). Three of these sequences can be interpreted in terms of stars pulsating in the fundamental mode

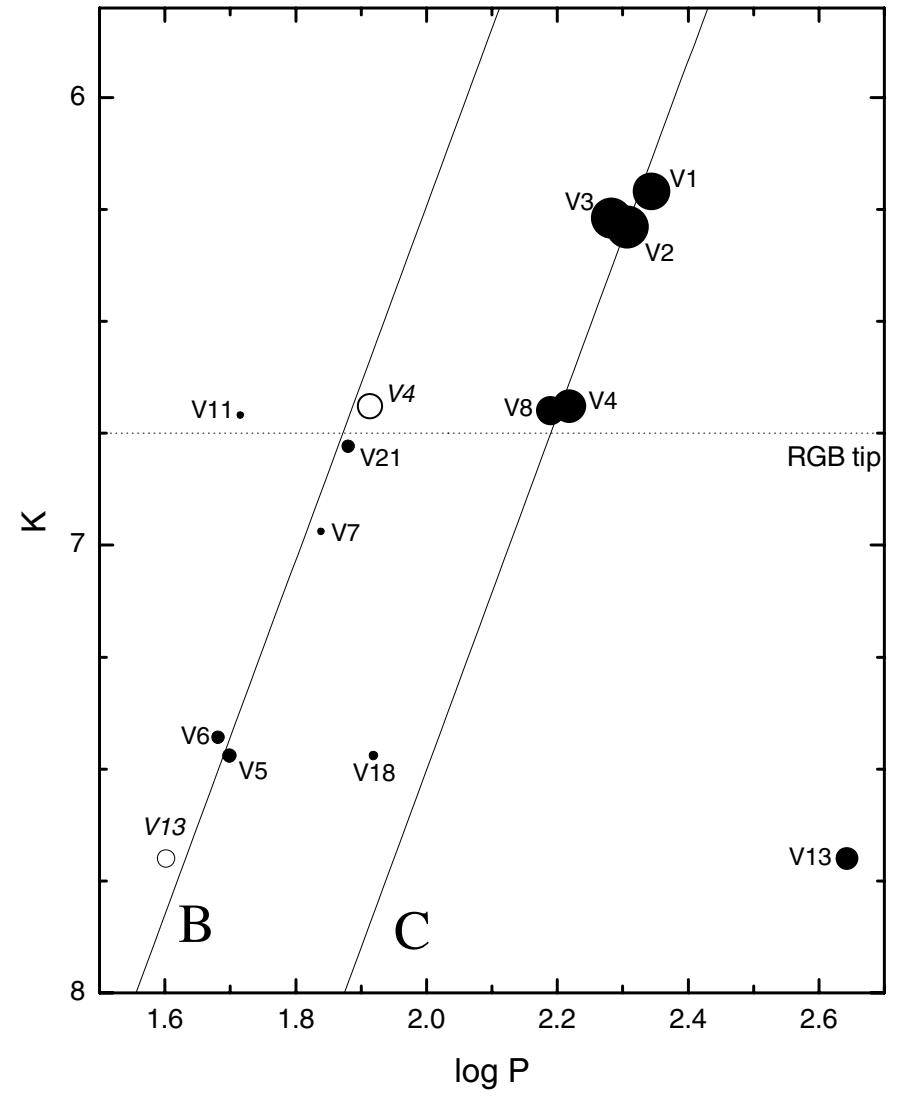

Fig. 14. $\log P$ vs. $K$ diagram for the long period variables in 47 Tuc. Symbol size denotes the velocity amplitude (ranging between 4 and $23 \mathrm{~km} \mathrm{~s}^{-1}$ ). Lines indicate the approximate location of sequences B and $\mathrm{C}$ found by Wood (2000) for LMC long period variables shifted to the distance of 47 Tuc. The dotted horizontal line marks the tip of the RGB according to Ferraro et al. (2000). Stars with possible multiple periods are shown twice.

and the first, second and third overtone modes ${ }^{2}$. The fourth period-luminosity relation still lacks a definite interpretation (Wood et al. 2004). For the Milky Way AGB variables the absence of reliable distances limits the establishment of such a period-luminosity relation (e.g. Bedding \& Zijlstra 1998). In the case of both the LMC and the Milky Way, the diversity of the stars in age, and therefore initial mass, complicates the interpretation.

The AGBs of globular clusters are characterized by a much more homogeneous set of stellar parameters. The richness of 47 Tuc in AGB variables allows us to use this advantage for a discussion of stellar pulsation in relation to luminosity, i.e. evolutionary stage. Period-luminosity diagrams for globular clusters have been constructed by several authors (e.g. Feast et al. 2002). Typically, only a relation for the large amplitude, long period variables ("Miras") was derived. This relation is in agreement with the sequence $\mathrm{C}$ found from $\mathrm{LMC}$ data (Wood 2000). In Fig. 14 we placed the AGB variables of 47 Tuc studied in this paper into a $\log P-K$-diagram. The approximate location of the $P-K$-relations $\mathrm{B}$ (first overtone) and $\mathrm{C}$

2 Sequence $\mathrm{A}$ is attributed to both second and third overtone mode pulsators. 
(fundamental mode) determined in the LMC (Wood 2000) are indicated by lines. Relations were transformed from the LMC to 47 Tuc distance using a LMC distance module of 18.515 (Clementini et al. 2003). Stars with more than one well established period in the literature or from our own light curve data are plotted twice and are marked accordingly in Table 2.

It can be seen that the variables nicely follow these two relations. The good fit of the $P-L$-relations to the 47 Tuc data after scaling from the LMC is indeed remarkable. Obviously, the physical mechanism that determines the location of at least these two sequences in the $\log P-K$-diagram is the same for LMC and 47 Tuc. Furthermore, the distance moduli we used in this paper seem to be very well determined at least concerning the relative distance between LMC and 47 Tuc. However, it has to be noted that the location of the sequences in the LMC is not accurately determined, as there is a significant scatter (probably due to the larger scatter in stellar parameters and maybe also the extension of the LMC) around the average locations used here. We therefore do not want to overinterpret our findings on that point.

There is a clear bipartition in our $\log P-K$-diagram (Fig. 14): at lower luminosities only sequence B is populated (see below for the case of V18), no variables are known in 47 Tuc that would fall on the lower part of $\log P-K$-relation $\mathrm{C}$. It is very likely that the stars V5, V6, V7, and V13, which we find in this lower luminosity part are probably in an earlier evolutionary state than the stars at higher luminosity (see below). In a first approach we assume that the luminosity of the stars in Fig. 14 corresponds to the evolutionary status.

At the highest luminosities only sequence $\mathrm{C}$ is occupied, and the stars there are the Miras V1, V2, and V3. Below these stars on sequence $\mathrm{C}$ lie V4 and V8. Both stars are located close to the tip of the RGB. They have similar velocity amplitude and $K$ brightness. V4 appears to switch modes between sequence $\mathrm{C}(P=165 \mathrm{~d})$ and sequence $\mathrm{B}(P=82 \mathrm{~d})$. From our data we have to conclude that V4 is currently only on sequence C. V11 having approximately the same $K$ luminosity as V4 is another star suspected of showing mode switching (see Sect. 4.1) located clearly off sequence B towards shorter periods (i.e. near sequence A). The period used in the plot is taken from Fox (1982). It is interesting that both stars with possible mode switching are found at similar $K$ luminosity, namely close to the RGB tip.

For V13 we found indications of a second period, and the star is therefore shown twice in Fig. 14. The long period of V13 is located close to sequence D from Wood (2000). The nature of this period-luminosity relation is not yet understood (Wood et al. 2004).

Symbol size in our $P-K$-diagram is linearly proportional to the measured velocity amplitude. In the case of V4 we stress again that the shorter period, marked with an open symbol in Fig. 14, is clearly not dominating the light or velocity variations at the moment. The symbol size of the V4 data point on sequence B may therefore be misleading as we have no indications that the velocity amplitude would be the same if the 82 days period would dominate. According to our data the star is currently monoperiodic. It can be seen that the large amplitude variables are all found along sequence C. Our results indicate further that the velocity amplitude is increasing along sequence C. Velocity amplitudes along sequence B are much smaller and do not show a steady increase of velocity amplitude with luminosity. The large amplitude of V13 seems to be associated with the long period ( $\sim 400 \mathrm{~d})$ variation rather than the $40 \mathrm{~d}$ variation of sequence $\mathrm{B}$.

These results lead us to the following probable evolutionary scenario: stars evolve up the AGB from low luminosities. They pulsate first in the first overtone (sequence B) then, at intermediate luminosities close to the RGB tip, they go through an interval of mode switching back and forth between first overtone and fundamental mode and finally, at the highest luminosities, they remain pulsating in the fundamental mode. There is clearly an increase in both the light and velocity amplitude associated with the switch from first overtone to fundamental. The large velocity amplitudes found at the tip of sequence $\mathrm{C}$ are similar to what is expected from models for fundamental mode pulsation (e.g. Scholz \& Wood 2000).

The role of V18 in this scenario is not clear. As noted in Sect. 3, this star has one of the largest infrared excesses among the 47 Tuc variables. In Fig. 14 we find the star in between sequences $\mathrm{B}$ and $\mathrm{C}$ at a rather low luminosity. Its velocity amplitude is rather small. However, locating this star on the period axis is difficult as the star has shown periodic, nonperiodic and constant phases in the past.

Comparing our results with evolutionary models requires some adaption of this scenario: due to luminosity variations during a Thermal Pulse the current location of a star in the $\log P-K$-diagram may not correspond to its evolutionary status along the AGB. However, as was shown e.g. by Boothroyd \& Sackmann (1988) the probability to observe the star at luminosity values highly deviating from the mean (and for the star's evolutionary status typical) value is comparatively low. This fact is even more expressed in stars of $1 M_{\odot}$ or less where the interpulse time is very large compared to the duration of the pulse itself (Vassiliadis \& Wood 1993). From the models of Vassiliadis \& Wood (1993, see their Fig. 13) for a star of $1 M_{\odot}$ and $Z=0.004$ we would expect to see about $1 / 6$ of our sample in a phase strongly deviating from the mean global parameters of the star, in our case these would be 2 stars (if our sample consists of AGB stars only, see Sect. 5.2). Most probably, these stars are expected to be found at a lower luminosity and a shorter period. It is therefore unlikely that one of the stars on sequence $\mathrm{C}$ belongs to this group. Following these considerations we have to add to our scenario the possible occurrence of loops in the $\log P-K$-diagram during the evolution up the AGB. V18 with its large IR excess and odd position in the $\log P-K$-diagram may be an example of such a star. However, it is unlikely that e.g. the mode switch of V4 is an indication of such a loop because of the time scale of the mode switch. Arp et al. (1963) gave a period of 165 days from data obtained in 1955/1956. Our light curve also favours this period. In between these two measurements are the light curve data presented by Fox (1982) giving the shorter period. The star therefore switched its pulsation mode back and forth within about 50 years. This seems to be hardly compatible with the longer timescales expected from evolutionary models. 
The mode switches seem to be concentrated in the luminosity range close to the RGB tip.

\section{2. $A G B$ or RGB stars?}

Throughout this paper we have assumed that all the variables discussed here are on the AGB. As can be seen from Fig. 14 a significant part of our sample is located below the tip of the red giant branch (RGB). The RGB tip plotted in Fig. 14 is taken from the recent study of Ferraro et al. (2000). They give a value of $K_{\mathrm{TRGB}}=6.75 \pm 0.2 \mathrm{mag}$. This result is in good agreement with the theoretical value expected for the metallicity of 47 Tuc (Salaris \& Cassisi 1998). All stars are above the low luminosity limit for thermally pulsing stars, so that they could belong to either the RGB or the AGB. The stars well above the RGB tip are clearly AGB stars, namely V1, V2, V3. V4 and V8 are both fundamental mode pulsators slightly above the RGB tip and thus they can be counted as AGB stars, too. For the other stars of our sample, we cannot decide on their AGB nature from luminosity alone. Colour information may be a good indicator for a separation, but our photometry is not homogeneous enough to decide on this rather small effect as a mean colour over the light cycle would be required.

Can variability properties allow us to distinguish between AGB and RGB stars? Using artificial luminosity functions, Alves et al. (1998) and Wood et al. (1999) argued that most pulsating stars below the RGB tip are in fact AGB stars. However, using data more sensitive to smaller amplitude variables, Ita et al. (2002) and Kiss \& Bedding (2003) have shown that a substantial fraction of variables below the RGB tip should be RGB stars.

According to the $\log P-K$-diagram for LMC variables given by Kiss \& Bedding (2003, see their Fig. 4), most of the variables detected below the tip of the RGB are pulsating in the second or third overtone mode. Furthermore, Kiss \& Bedding split their $\log P-K$-diagram into six parts according to the light amplitude in $I$. Most of the variables below the tip of the RGB show amplitudes between 0.01 (their detection limit) and $0.14 \mathrm{mag}$. The luminosity function for these stars indicates a substantial fraction are on the RGB. However, for larger light amplitudes, the RGB component is no longer detectable. Kiss $\&$ Bedding also give colour information showing that by far the largest fraction of stars below the RGB tip with amplitudes $>0.14 \mathrm{mag}$ is found on the blue side of the red giant branch, which again favours attributing these stars to the AGB. Thus larger light amplitudes seem an indication of AGB status.

Kiss \& Bedding (2003) used $I$ band data, whereas we have $V$ band light curves. Fox (1982) presents $V$ and $I$ light curves for several of our sample stars. For the stars of lower luminosity like V5 or V6, the $I$ amplitude is about a factor of 2 less than the $V$ amplitude. The $V$ band amplitudes of the stars of our sample are all $\geq 0.2 \mathrm{mag}$, i.e. we estimate an $I$ amplitude of $\geq 0.1 \mathrm{mag}$.

V21, V5 and V6 all show light amplitudes of several tenth of a magnitude. Such amplitude values favour an AGB nature for these stars as well. V7 shows a rather small light amplitude, and for V11 the parameters of the light change are not clear. Therefore, we cannot decide if these two objects are RGB or
AGB stars. Interestingly, both objects also have smaller velocity amplitudes.

Another unclear case is V13. The total amplitude measured by us is $\approx 0.7 \mathrm{mag}$. But this amplitude is dominated by the long period variation, the short period variation has a much smaller amplitude. The amplitudes used in the discussion by Kiss \& Bedding are derived from Fourier analysis of the individual frequency components. Therefore the amplitude of the short period is the relevant one in our argumentation. As a result V13 may well be a RGB star.

Our approach is based on statistical arguments and not on a complete understanding of RGB pulsation. In particular, we note that the LMC stars of Kiss \& Bedding (2003) are likely to be more massive than the 47 Tuc stars and their amplitude behaviour may be quite different to that of 47 Tuc stars. We therefore cannot exclude the possibility that some of the variables below the RGB tip are on the RGB, but for many of them a location on the AGB seems to be more likely.

\subsection{Comparison with mass loss}

Using the velocity amplitude we can separate the $\log P-K$-diagram (Fig. 14) of 47 Tuc into three to four regions. At highest luminosity we find the Mira variables (V1, V2 and V3) with the largest velocity amplitudes. Going down sequence $\mathrm{C}$, we find the second group (V4 and V8) with intermediate amplitudes. The third group consists of stars with low velocity amplitude on sequence B (and possibly A). Finally, V13 may form a special case with its large amplitude due to a long secondary period.

Mass loss data available in the literature are limited to measurements of circumstellar dust. As there may be a dust-free mass loss as well, e.g. during the RGB phase, we cannot rule out that stars with indications for no or only small amounts of circumstellar dust still have a considerable mass loss rate. However, it is very likely that the existence of dust enhances the mass loss rate (e.g. Höfner et al. 1996), so we expect that the infrared excess is at least a measure for the relative mass loss rate within the cluster. As a caveat we have to mention the lack of infrared photometry beyond $12 \mu \mathrm{m}$ for the stars of our sample. This certainly limits the reliability of estimates for the amount of dust surrounding a star.

Note that the circumstellar material currently around each star may originate from a time when the star's pulsation was different. Another factor to keep in mind is that it is well known (e.g. Olofsson et al. 2000) that, at least in some cases, mass loss in AGB stars may be episodic rather than continuous. This has also been noted in the context of globular clusters by Origlia et al. (2002).

According to Reimers' (1975) law, we would expect the mass loss rate to increase with luminosity. Therefore it is difficult to separate the effects of luminosity and pulsation (velocity amplitude) on the mass loss rate: high mass loss rates are found at high luminosities, where we also find large velocity amplitudes. We can, however, try to compare mass loss rate and velocity amplitude for some stars of similar luminosity. Such a group of stars is found around the RGB tip (V8, V4, 
V11 and V21). Origlia et al. (2002) measure $K-[12]$ values for V8 and V21. V8 shows a clear dust excess while V21 does not. Comparing the velocity amplitudes for these two stars shows that V8 also has a significantly larger velocity amplitude than V21. V4 is also a star with an infrared dust excess (Frogel \& Elias 1988). It has a similar velocity amplitude as V8. Gillett et al. (1988) report an infrared excess for V11, but a separation between V11 and the nearby variable V18 is very difficult on the IRAS images they used. Ramdani \& Jorissen (2001) later found an infrared excess of V18 from ISO observations, while V11 has only a very small infrared excess. We conclude that, at similar luminosity, stars with higher pulsation amplitudes show higher mass loss rates.

V5, V6 and V18 form a second group of variables at similar luminosity. Unlike the group of stars around the RGB tip, these stars all have very similar velocity amplitudes. Neither V5 nor V6 has an infrared excess reported in the literature. But V18 has an outstanding infrared excess according to Ramdani \& Jorissen (2001). As noted above, the high infrared excess of V18 at a relatively low luminosity suggests that this star is currently in the luminosity minimum following a thermal pulse on the AGB.

V13, the star with the long secondary period, shows no indication of circumstellar dust. It appears that these long periods on sequence $\mathrm{D}$ are not directly related to a mass loss phenomenon (compare also Wood et al. 2004).

Origlia et al. (2002) detected five further stars nearer to the core of 47 Tuc that show considerable infrared excesses but that are not known to be variable star. We checked our photometric monitoring data covering two months in late 2003 for variable stars at the positions given by Origlia et al. Four of the five stars had variable counterparts on our $V$ frames. The fifth object is the one with the lowest mass loss rate in the list of Origlia et al. All four objects varied during the two months by several tenths of a magnitude. More detailed results on these stars including period determination will be presented in a forthcoming paper (Lebzelter \& Wood in prep.).

Summarizing our results regarding the correlation of mass loss and velocity amplitude: stars with significant mass loss are all pulsating, a fact already noted by other authors (e.g. Ramdani \& Jorissen 2001). Stars on sequence C combine large velocity amplitudes with comparably high mass loss rates. On sequence B all stars have low velocity amplitudes and most of them also have no or very modest mass loss. For stars of similar luminosity, increased pulsational amplitude seems to significantly increase the mass loss rate. One star (V18) currently showing small pulsation amplitude has considerable amounts of circumstellar dust, presumably from a past interval of higher amplitude pulsation. We speculated that V18 is currently doing a loop in the $\log P-K$-diagram as a consequence of a recent or ongoing thermal pulse. Its circumstellar dust may then originate not only from a phase of higher amplitude pulsation but also from a time the star was more luminous.

Acknowledgements. T.L. has been supported by the Austrian Academy of Science (APART programme). P.R.W. has been partially supported by a grant from the Australian Research Council. This research at Tennessee State University was partially funded by
NASA grant NCC5-511 and NSF grant HRD-9706268. This publication makes use of data products from the Two Micron All Sky Survey, which is a joint project of the University of Massachusetts and the Infrared Processing and Analysis Center/California Institute of Technology, funded by the National Aeronautics and Space Administration and the National Science Foundation. Partly based on observations obtained at the Gemini Observatory, which is operated by the Association of Universities for Research in Astronomy, Inc., under a cooperative agreement with the NSF on behalf of the Gemini partnership: the National Science Foundation (US), the Particle Physics and Astronomy Research Council (UK), the National Research Council (Canada), CONICYT (Chile), the Australian Research Council (Australia), CNPq (Brazil), and CONICRT (Argentina). Partly based on observations obtained with the Phoenix infrared spectrograph, developed and operated by the National Optical Astronomy Observatory. We wish to thank the referee Jacco van Loon for his helpful comments.

\section{References}

Allen, D. A., \& Cragg, T. A. 1983, MNRAS, 203, 777

Alves, D., Alcock, C., Cook, K., et al. 1998, in Pulsating Stars: Recent Developments in Theory and Observation, ed. M. Takeuti, \& D. D. Sasselov (Tokyo: Universal Academic Press), 17

Alves, D. R., Bond, H. E., \& Livio, M. 2000, AJ, 120, 2044

Arp, H. C., Brueckel, F., \& Lourens, J. V. B. 1963, ApJ, 137, 228

Bates, B., Catney, M. G., \& Keenan, F. P. 1990, MNRAS, 245, 238

Bedding, T. R., \& Zijlstra, A. A. 1998, ApJ, 506, L47

Bertelli, G., Bressan, A., Chiosi, C., Fagotto, F., \& Nasi, E. 1994, A\&AS, 106, 275

Boothroyd, A. I., \& Sackmann, I.-J. 1988, ApJ, 328, 632

Bowen, G. H., \& Willson, L. A. 1991, ApJ, 375, L53

Briley, M. M., Smith, V. V., King, J. R., \& Lambert, D. L. 1995, Nature, 383, 604

Caretta, E., \& Gratton, R. G. 1997, A\&AS, 121, 95

Carpenter, J. 2000, www.astro.caltech.edu/ jmc/2mass/ v3/transformations/

Clement, C. M., Muzzin, A., Dufton, Q., et al. 2001, AJ, 122, 2587

Clementini, G., Gratton, R., Bragaglia, A., et al. 2003, AJ, 125, 1309 Cohen, J. 1976, ApJ, 203, L27

Evans, A., Stickel, M., van Loon, J. T., et al. 2003, A\&A, 408, L9

Feast, M., Whitelock, P., \& Menzies, J. 2002, MNRAS, 329, L7

Ferraro, F. R., Montegriffo, P., Origlia, L., \& Fusi Pecci, F. 2000, AJ, 119,1282

Forte, J. C., Bassino, L. P., Vega, E. I., et al. 2002, AJ, 123, 3263

Frogel, J. A. 1983, ApJ, 272, 167

Frogel, J. A., \& Elias, J. H. 1988, ApJ, 324, 823

Frogel, J. A., Persson, S. E., \& Cohen, J. G. 1981, ApJ, 246, 842

Fox, M. W. 1982, MNRAS, 199, 715

Gillett, F. C., de Jong, T., Neugebauer, G., Rice, W. L., \& Emerson, J. P. 1988, AJ, 96, 116

Glass, I. S., \& Feast, M. W. 1973, MNRAS, 163, 245

Gratton, R. G., Bragaglia, A., Carretta, E., et al. 2003, A\&A, 408, 529

Helling, Ch., Arnst, T. U., \& Sedlmayr, E. 2002, in Radial and Nonradial Pulsations as Probes of Stellar Physics, ASP Conf. Ser., 259, ed. C. Aerts, T. Bedding, \& J. Christensen-Dalsgaard, 546

Hesser, J. E., Harris, W. E., Vandenbergh, D. E., et al. 1987, PASP, 99, 739

Hinkle, K. H. 1978, ApJ, 220, 210

Hinkle, K. H., Hall, D. N. B., \& Ridgway, S. T. 1982, ApJ, 252, 697

Hinkle, K. H., Lebzelter, T., \& Scharlach, W. W. G. 1997, AJ, 114, 2686

Höfner, S., Feuchtinger, M. U., \& Dorfi, E. A. 1996, A\&A, 297, 815 
Ita, Y., Tanabe, T., Matsunaga, N., et al. 2002, MNRAS, 337, L31

Joyce, R. R., Hinkle, K. H., Meyer, M. R., \& Skrutskie, M. F. 1998, Proc. SPIE, 3354, 741

Kiss, L. L., \& Bedding, T. R. 2003, MNRAS, 343, L79

Lebzelter, T., \& Hinkle, K. H. 2002, in Radial and Nonradial Pulsations as Probes of Stellar Physics, ASP Conf. Ser., ed. C. Aerts, T. Bedding, \& J. Christensen-Dalsgaard, 259, 556

Lebzelter, T., Kiss, L. L., \& Hinkle, K. H. 2000, A\&A, 361, 167

Lee, S. W. 1977, A\&AS, 27, 381

Lloyd Evans, T. 1974, MNRAS, 167, 393

Lloyd Evans, T., \& Menzies, J. W. 1973, in Variables Stars in Globular Clusters and in Related Systems, ed. J. D. Fernie (ReidelDordrecht), 151

Lyons, M. A., Kemp, S. N., Bates, B., \& Shaw, C. R. 1996, MNRAS, 280,835

Menzies, J. W., \& Whitelock, P. A. 1985, MNRAS, 212, 783

Montegriffo, P., Ferraro, F. R., Fusi Pecci, F., \& Origlia, L. 1995, MNRAS, 276, 739

Olofsson, H., Bergman, P., Lucas, R., \& Eriksson, K. 2000, A\&A, 353,583

Origlia, L., Scaltriti, F., Anderlucci, E., et al. 1997, MNRAS, 292, 753

Origlia, L., Ferraro, F. R., Fusi Pecci, F., \& Rood, R. T. 2002, ApJ, 571,458

Ramdani, A., \& Jorissen, A. 2001, A\&A, 372, 85
Reimers, D. 1975, Mem. Soc. Roy. Sc. Liege, 6e Ser., 8, 369

Salaris, M., \& Cassisi, S. 1998, MNRAS, 298, 166

Sawyer-Hogg, H. 1973, Publ. DDO, 3, No. 6

Scholz, M., \& Wood, P. R. 2000, A\&A, 362, 1065

Schröder, K.-P., \& Sedlmayr, E. 2001, A\&A, 366, 913

Soker, N., Catelan, M., Rood, R. T., \& Harpaz, A. 2001, ApJ, 563, L69

Udry, S., Mayor, M., Maurice, E., et al. 1999, in Precise Stellar Radial Velocities, ASP Conf. Ser., 185, IAU Coll., 170, ed. J. B. Hearnshaw, \& C. D. Scarfe, 383

van Loon, J. T., Groenewegen, M. A. T., de Koter, A., et al. 2001, A\&A, 351, 559

Vassiliadis, E., \& Wood, P. R. 1993, ApJ, 413, 641

Whitelock, P. A. 1986, MNRAS, 219, 525

Wood, P. R. 1979, ApJ, 227, 220

Wood, P. R., Whiteoak, J. B., Hughes, S. M. G., et al. 1992, ApJ, 397, 552

Wood, P. R., \& the MACHO Collaboration 1999, in IAU Symp., 191, Asymptototic Giant Branch Stars, ed. T. Le Bertre, A. Lèbre, \& C. Waelkens (San Francisco: ASP), 151

Wood, P. R. 2000, PASA, 17, 18

Wood, P. R., Olivier, E., \& Kawaler, S. 2004, ApJ, 604, 800

Zinn, R., \& West, M. J. 1984, ApJS, 55, 45 